By writing $y$ for $\pi / \sqrt{ } x$ in the above relations, we obtain a corresponding set of expressions for $\sum_{n=1}^{\infty} \Phi_{2 r+1}(n y)$ for $y \geqq 10$. For example from (8) we have

$$
\sum_{n=1}^{\infty} \Phi_{2 r+1}(n y) \doteqdot(-1)^{r+1}(2 r) ! / \pi^{\frac{1}{2}} r !
$$

which is independent of $y(\geqq 10)$, and for $r=1$ gives

$$
\sum_{n=1}^{\infty} e^{-\frac{1}{2} n^{n} z^{2}} D_{2}(n z) \doteq \frac{1}{2}, \text { if } z>14 \text {. }
$$

Gairview, Glencairn St., Stevenston, Ayrshire.

\title{
A Generalisation of Dirichlet's Multiple Integral
}

\author{
By I. J. Good.
}

The purpose of this note is to generalise the Dirichlet-Liouville formula which expresses a certain type of multiple integral in terms of a single integral. ${ }^{1}$ In our formula the multiple integral will involve several arbitrary functions instead of only one, and it will be expressed as a product of single integrals.

Let $n$ be a positive integer. Let $f_{1}(t), f_{2}(t), \ldots, f_{n}(t)$ be Lebesgue measurable functions when $0 \leqq t \leqq 1$. A finite sequence of $n$ real numbers $m_{1}, m_{2}, \ldots, m_{n}$ is given. We write $m_{n+1}=0$ and

$$
\begin{aligned}
& M_{i}=m_{1}+m_{2}+\ldots+m_{r} \\
& X_{r}=x_{1}+x_{2}+\ldots+x_{r}
\end{aligned}
$$

1 See, for example, G. F. Meyer, Vorlesungen über die Theorie der bestimmten Integrale (Leipzig, 1871), 566 et seq.; or E. T. Whittaker and G. N. Watson, Modern Analysis (4th edn., Cambridge, 1935), section 12.5; or H. Jeffreys and B. S. Jeffreys, Methods of Mathematical Physics (Cambridge, 1946), section 15.08; or L. J. Mordell, “ Dirichlet's integrals," Edin. Math. Notes, No. 34 (1944), 15-17. 
and so on, and we assume that all variables of integration, such as $x_{1}, x_{2}, \ldots, x_{n}$, are non-negative. Then

$$
\begin{gathered}
\int_{X_{n} \leqslant 1} \ldots \int_{r=1}^{n-1} \prod_{r}^{m_{r}} f_{r}^{m_{r}}\left(\frac{X_{r}}{X_{r+1}}\right) x_{n}^{n_{n}} f_{n}\left(X_{n}\right) d x_{1} d x_{2} \ldots d x_{n} \\
=\prod_{r=1}^{n} \int_{0}^{1} f_{r}(x)(1-x)^{n_{r}+1} x^{M_{r}+r-1} d x
\end{gathered}
$$

provided that the $n$ single integrals on the right all exist.

Proof. We proceed in a formal spirit. The proof can easily be made rigorous by working backwards from the final result and making use of Fubini's theorem.

Denote the left-hand side of (1) by $I_{n}\left(f_{1}, f_{2}, \ldots, f_{n}\right)$. In this $n$-fold integral wo first change the variables of integration from $x_{1}, x_{2}, \ldots, x_{n}$ to $x_{1}, x_{2}, \ldots, x_{n-1}, X_{n}$ and second put $x_{r}=X_{n} y_{r}(r=1,2$, $\ldots, n-1)$. We obtain $I_{n}\left(f_{1}, f_{2}, \ldots, f_{n}\right)$

$$
\begin{aligned}
& =\int_{0}^{1} f_{n}\left(X_{n}\right) d X_{n} \int_{X_{n-1} \leq X^{n}} \ldots \int_{n}\left(X_{n}-X_{n-1}\right)^{m_{n}} \prod_{r=1}^{n-1}\left\{x_{r}^{m_{r}} f_{r}\left(\frac{X_{r}}{X_{r+1}}\right) d x_{r}\right\} \\
& =\int_{0}^{1} f_{n}\left(X_{n}\right) X_{n}{ }^{1 I_{n}+n-1} d X_{n} \int \ldots \int_{r=1}^{n} \prod_{Y_{n-1}<1}^{2}\left\{y_{r}^{m_{r}} f_{r}\left(\frac{Y_{r}}{Y_{r+1}}\right)\right\} \\
& y_{n-1}^{m_{n-1}} f_{n-1}\left(Y_{n-1}\right)\left(1-Y_{n-1}\right)^{m_{n}} d y_{1} \ldots d y_{n-1}
\end{aligned}
$$

$=I_{n-1}\left(f_{1}, f_{2}, \ldots, f_{n-2}, g_{n-1}\right) \int_{0}^{1} f_{n}(x) x^{H_{n}+n-1} d x$,

where $g_{n-1}(t)=f_{n-1}(t)(1-t)^{m_{n}}$. The theorem now follows by induction.

As a special case let $f_{r}(t)=t \wedge_{r}$ where $\Lambda_{r}=\lambda_{1}+\lambda_{2}+\ldots+\lambda_{r}$ and assume $m_{r}>-1, \lambda_{r}+m_{r}>-1$. Then

$$
\int_{X_{n} \leq 1} \ldots \int_{r=1}^{n}\left\{x_{r}^{m_{r}} X_{r}^{\lambda_{r}} d x_{r}\right\}=\prod_{r=1}^{n} \frac{\Gamma\left(\Lambda_{r}+M_{r}+r\right) \Gamma\left(m_{r+1}+1\right)}{\Gamma\left(\Lambda_{r}+M_{r+1}+r+1\right)}
$$

where $m_{n+1}=0$.

131 Cheviot Gardens,

LoNDON, N.W.2. 